\title{
Insieme a distanza. Alleanze educative tra servizi per l'infanzia e famiglie durante il lockdown
}

\author{
Maja Antonietti, Monica Guerra ${ }^{1}$,Elena Luciano ${ }^{3,4}$
}

\begin{abstract}
La relazione con le famiglie costituisce un tema fondativo nei servizi educativi 0-6. Le istanze partecipative e inclusive che, da sempre, contraddistinguono in Italia i servizi per l'infanzia, sono state messe fortemente in discussione durante la pandemia da Covid-19 perché l'emergenza sanitaria, sociale, politica ed economica ha modificato profondamente tempi, spazi e modi della comunicazione e delle relazioni, dentro e fuori i servizi e le scuole per l'infanzia. Il presente contributo analizza il tema della relazione tra servizi e famiglie restituendo e discutendo i dati emergenti da una ricerca esplorativa svolta in merito all'esperienza di educazione a distanza durante la fase di lockdown in Italia nell'ambito dei servizi per l'infanzia dalla nascita ai sei anni. Attraverso un questionario sono state raccolte le opinioni di un campione di educatori, insegnanti e coordinatori in merito alle pratiche adottate relativamente ai legami educativi a distanza (LEAD) e, tra le varie dimensioni esplorate, vengono qui problematizzati i modi e le forme attraverso cui la partecipazione delle famiglie si è trasformata nella distanza.
\end{abstract}

Parole chiave: inclusione, famiglie; servizi di educazione e cura dell'infanzia 0-6, Covid-19, coeducazione.

\section{Abstract}

The relationship with families is a crucial topic in educational services for children aged 0-6 years. The participatory and inclusive approach that has traditionally characterized

${ }^{1}$ Ricercatrice in Didattica e pedagogia speciale presso il Dipartimento di Discipline Umanistiche, Sociali e delle Imprese Culturali dell'Università degli Studi di Parma.

${ }^{2}$ Professoressa associata di Pedagogia generale e sociale presso il Dipartimento di Scienze Umane per la formazione "Riccardo Massa" dell'Università degli Studi di Milano-Bicocca.

${ }^{3}$ Professoressa associata di Pedagogia generale e sociale presso il Dipartimento di Discipline Umanistiche, Sociali e delle Imprese Culturali dell'Università degli Studi di Parma.

${ }^{4} \mathrm{Il}$ carattere e i contenuti del presente contributo sono stati condivisi dalle tre Autrici; i parr. 2 e 3 sono attribuibili a Maja Antonietti, i parr. 4 e 5 a Monica Guerra e il par. 1 a Elena Luciano (d'ora in poi, laddove non diversamente specificato, le note a piè di pagina si intendono a cura delle Autrici, N.d.R.). 
Italian early childhood education and care has come under serious pressure during the Covid-19 pandemic: the ongoing health, social, political, and economic emergency has radically modified timeframes, spaces, an modes of communicating and relating, both in early years/nursery school settings and more generally. This paper examines the relationship between early childhood education services and families, by reporting and analyzing data from an exploratory study on distance education in services for children aged 0-6 years, during the spring 2020 lockdown in Italy. Specifically, a questionnaire was used to collect the views of a sample of educators, teachers, and coordinators concerning the practices that had been implemented in support of the remote educational relationship (in Italian, "Legami Educativi A Distanza - LEAD” programme). Among the various themes investigated, the focus here is on problematizing the ways in which families' participation changed during the distance education phase.

Keywords: inclusion, families, Early Childhood Education and Care services, Covid-19, coeducation.

\section{La relazione con le famiglie in tempi di pandemia}

La relazione con le famiglie è riconosciuta, a livello internazionale, come un tema cruciale nell'ambito del sistema dei servizi educativi per l'infanzia. Essa costituisce infatti un criterio di qualità imprescindibile su cui è opportuno fare continuamente leva, come è evidenziato in una pluralità di contributi e documenti di indirizzo tra i quali, per esempio, diversi studi pubblicati dall'OCSE, compresa la serie di report Starting Strong pubblicati tra il 2001 e il 2017, che hanno posto la partecipazione delle famiglie e delle comunità tra le dimensioni strategiche utili a promuovere inclusione sociale e partecipazione democratica (OECD, 2012).

Se la partecipazione delle famiglie nei servizi facilita infatti l'ambientamento, le conquiste e gli apprendimenti dei bambini (Harris, Goodall, 2006; Powell et al., 2010; Weiss et al., 2008), essa trova senso dentro un'ampia sinergia tra i servizi per l'infanzia, le famiglie, il vicinato e tutti gli attori della società civile che può offrire risposte più aderenti ai bisogni e alle potenzialità dei bambini e di tutte le famiglie, comprese quelle in situazioni di svantaggio sociale (OECD, 2012). È utile considerare, a tal proposito, che la ricerca scientifica, nel tempo, ha mostrato tutto il valore della partecipazione dei genitori all'interno dei servizi e delle scuole per l'infanzia e ha favorito il suo passaggio da mezzo strumentale al successo scolastico dei bambini a opportunità di apprendimento e di crescita ad ampio raggio (Brougère, 2009), per i bambini, per gli adulti e per le istituzioni coinvolte.

In tal senso, il contributo della teoria dell'ecologia dello sviluppo umano (Bronfenbrenner, 1979, trad. it. 1986) è stato particolarmente ri- 
levante nel mostrare l'impatto delle relazioni tra famiglie e servizi/scuole sullo sviluppo complessivo dei bambini, fino a diffondere a livello internazionale la consapevolezza della necessità di accogliere, insieme al bambino, anche le sue figure familiari.

In ambito europeo, anche la proposta di principi chiave per la qualificazione dei servizi per l'infanzia da parte del Gruppo di Lavoro Tematico sull'Educazione e Cura dell'Infanzia sotto l'egida della Commissione Europea ha fortemente valorizzato «il coinvolgimento attivo delle famiglie, volto a rilanciare la progettualità dei servizi e delle scuole dell'infanzia in una prospettiva che valorizza la diversità socio-culturale, ovvero l'iperdiversità attuale, che tanto caratterizza ogni contesto in Italia come negli altri paesi europei» (trad. it. a cura di Lazzari, 2016, p. 7). In relazione a ciò, il secondo dei 10 principi individuati dalla Commissione Europea promuove proprio servizi che incoraggino la partecipazione, rafforzino l'inclusione sociale e accolgano la diversità, attraverso:

un approccio collaborativo alla promozione dei benefici derivanti dalla frequenza dei servizi che coinvolga le comunità locali e le agenzie territoriali; approcci che rispettino e valorizzino le convinzioni, i bisogni e la cultura delle famiglie di provenienza; la garanzia che tutti i bambini e i loro genitori siano accolti all'interno del servizio, un approccio che incoraggia intenzionalmente e attivamente tutti i genitori all'utilizzo dei servizi; il riconoscimento che il personale che opera all'interno di tali servizi dovrebbe essere formato a rendere i genitori e le famiglie consapevoli del valore dei servizi, garantendo loro che le proprie convinzioni e la propria cultura saranno rispettate - questa formazione potrebbe essere supportata in modo complementare anche dall'attivazione di programmi a sostegno della genitorialità; una stretta collaborazione tra il personale che opera nei servizi per l'infanzia e il personale che opera nei servizi sociosanitari e scolastici a livello territoriale (Commissione Europea, 2016, p. 25).

In Italia, in particolare, la partecipazione delle famiglie costituisce un tema fondativo nei servizi educativi 0-6. La storia stessa dei servizi per l'infanzia italiani è infatti densa di istanze partecipative e di movimenti dal basso. È una storia animata da donne e uomini che, nel secondo dopoguerra, hanno iniziato a contribuire alla costruzione - edilizia, sociale, educativa e culturale - di scuole per i bambini e le bambine capaci di promuovere diversità, risorse e talenti per costruire libertà e emancipazione (Bove, 2003; Campioni, Marchesi, 2014; Edwards, Gandini, Forman, 1995).

Se, dal punto di vista storico, i servizi e le scuole per l'infanzia italiani si contraddistinguono proprio per i processi partecipati che - a diversi 
livelli - hanno coinvolto e coinvolgono i vari attori che li abitano, anche dal punto di vista pedagogico e progettuale oggi l'investimento sulla relazione con le famiglie nei servizi e nella scuola per l'infanzia - in una logica di coeducazione, di partnership e di alleanza educativa (Bove, 2020; Guerra, Luciano, 2009, 2014; Milani, 2008, 2018; Pileri, 2017; Pourtois, Desmet, 2015; Rayna, Rubio, Scheu, 2010) - risulta irrinunciabile, come del resto proposto e promosso nell'ambito delle Indicazioni Nazionali del 2012, della normativa vigente in materia di istituzione del sistema integrato di educazione e istruzione dalla nascita ai sei anni (L.107/2015; D.lgs. 65/2017), nonché nel vivo di moltissimi progetti pedagogici e piani triennali dell'offerta formativa diffusi su tutto il territorio nazionale.

Durante l'atroce pandemia da Covid-19, le istanze partecipative e inclusive che, da sempre, contraddistinguono i servizi per l'infanzia sono state messe fortemente in discussione perché la diffusa emergenza sanitaria, sociale, politica ed economica, travolgendo il mondo intero, ha anche modificato profondamente tempi, spazi e modi della comunicazione e delle relazioni dentro e fuori i servizi educativi e scolastici 0-6, ovvero sia all'interno delle singole famiglie e delle reti intra- e inter-familiari, sia nel funzionamento dei servizi educativi e delle scuole per l'infanzia sia nelle reti territoriali entro le quali essi sono situati.

Fin dal lockdown del marzo 2020, la nascita di pratiche educative in modalità a distanza, finalizzate a mantenere vivi i legami educativi con i bambini e le famiglie frequentanti i servizi e le scuole per l'infanzia, ha posto immediatamente in chiaro che - senza il coinvolgimento e il supporto delle famiglie stesse - nessun Legame Educativo A Distanza (LEAD) (MIUR, 2020a) era possibile. In quella prima fase di dolore e disorientamento collettivo, erano proprio le figure familiari gli unici mediatori possibili di una qualsiasi forma di relazione educativa ideata e promossa dal servizio e concessa dalla tecnologia.

Tuttavia, al di là di questa prima, fondamentale funzione strumentale che la famiglia ha assunto per l'avvio dei LEAD, le relazioni tra educatori e figure familiari in molte realtà educative si sono progressivamente trasformate, talora ancorandosi a un'alleanza educativa profonda e promossa perché considerata cruciale già prima della pandemia.

Proprio in tale direzione si è orientato l'investimento sulla relazione con le famiglie promosso all'interno degli orientamenti pedagogici dei Legami Educativi A Distanza (LEAD) in tempi di pandemia pubblicato a maggio 2020 dalla Commissione per il Sistema integrato di educazione e di istruzione dalla nascita ai sei anni «per supportare gli operatori dei nidi e delle scuole dell'infanzia nell'opera di rinsaldamento delle relazio- 
ni educative con i bambini del nido e della scuola dell'infanzia e con i loro genitori durante e dopo la fase emergenziale legata alla pandemia» ${ }^{5}$. All'interno del documento ministeriale, è infatti evidente l'obiettivo di rinegoziare il patto educativo tra personale educativo e figure familiari, in una logica di inclusione, di attiva collaborazione, di co-progettazione e di partnership educativa:

Con le videochiamate le insegnanti entrano nelle case dei bambini, vedono frammenti di luoghi e atmosfere, intessono relazioni con chi nella casa abita, colgono alcune pratiche genitoriali di cura, di relazione, di promozione dell'autonomia dei bambini. Al tempo stesso le educatrici sono osservate dai genitori nella relazione che instaurano con il loro bambino e con $\mathrm{i}$ bambini in gruppo. I LEAD richiedono necessariamente la mediazione dei genitori, i quali - ancor più che nella scuola in presenza - assumono un ruolo attivo di partner educativi, a partire dalla progettazione del momento dell'incontro. Alle insegnanti, professioniste dell'educazione, vengono richieste sensibilità e apertura al dialogo e al confronto, ai genitori vengono richiesti rispetto dei ruoli e collaborazione attiva. Non è opportuno da parte delle insegnanti pensare che i genitori debbano, possano e vogliano ricostruire l'ambiente e la giornata educativa del nido o della scuola in ambito domestico; al contempo non è praticabile da parte dei genitori delegare la gestione dei bambini all'educatrice per il tempo del collegamento in video, aspettandosi un mero intrattenimento a distanza. È opportuno, invece, rinegoziare spazi e tempi, entrare nelle case "in punta di piedi" e rispettarne l'intimità e le complessità portate dall'eventuale smart working dei genitori, concordare i momenti dell'incontro e della separazione, individuare insieme gli strumenti e le proposte più accessibili e più gradite (MIUR, 2020a, p.3).

Oggi, a molti mesi di distanza dall'inizio della pandemia e tuttora in fase di emergenza, sia la realtà delle famiglie sia quella dei servizi educativi e scolastici rivolti ai bambini di età 0-6 e alle loro famiglie sono messe a dura prova sul piano economico, sociale, psicologico, culturale e organizzativo e l'invito di Cagliari (2013) di alcuni anni fa, risulta oggi quanto mai attuale:

è necessario ricostruire una grammatica dei servizi educativi $0 / 6$ che ridefinisca la loro identità proprio a partire dalla partecipazione della società civile e delle famiglie al progetto educativo, gestionale, organizzativo, culturale e politico del nido e della scuola dell'infanzia (Cagliari, 2013, p. 6).

${ }^{5}$ https://www.miur.gov.it/web/guest/-/orientamenti-pedagogici-sui-led (data di ultima consultazione: 4.3.21). 
Dagli esiti di un progetto di ricerca-azione svolta durante il lockdown nell'ambito del progetto internazionale RICE (Rete Internazionale delle Città dell'Educazione), volta a indagare la riorganizzazione delle vite familiari e professionali di genitori e personale educative e le prassi di coeducazione promosse nell'emergenza (Pileri, 2020), emerge dai genitori coinvolti in alcuni focus group l'idea che il forzato confinamento abbia valorizzato particolarmente il ruolo di nidi e scuole e abbia reso urgente il bisogno di sentirsi da essi coinvolti e supportati attraverso nuove forme di relazione, non lasciando indietro nessuno:

Accanto alle preoccupazioni è possibile cogliere anche la volontà di tornare alla normalità, di stare in rete fra scuola-famiglie-comunità, di pensare a nuove forme di coinvolgimento e di alleanza anche fra istituzioni, di trovare il modo per non lasciare nessuno indietro, di cogliere questa occasione per migliorare criticità, valorizzare e diffondere aspetti di qualità mettendoli a sistema, come nel caso della nascita di nuove forme di solidarietà cittadina (Ivi, p. 86).

Insegnanti e coordinatori coinvolti nella medesima ricerca hanno confermato, del resto, la necessità di trovare nuove forme di coinvolgimento delle famiglie, anche a fronte della difficoltà di partecipazione di alcune di esse, e una cura specifica relativamente alla comunicazione e all'inclusione di tutte le famiglie, comprese quelle migranti e di quelle con bisogni educativi speciali (Ibidem, passim).

Alcune indagini sulla quotidianità delle famiglie durante l'avvio dell'emergenza sanitaria hanno evidenziato, nonostante la drammaticità della situazione, risvolti costruttivi e insegnamenti tratti in essa dai genitori, come l'apprezzamento di relazioni sociali e aspetti della quotidianità dati prima per scontati e di atteggiamenti ispirati a collaborazione, solidarietà e senso civico (Gigli, 2020).

La voce dei genitori è stata raccolta anche nell'ambito dell'indagine Bambini e lockdown: la parola ai genitori, promossa dalla Società Italiana dei Pediatri delle Cure Primarie Pediatriche (SICuPP-Lombardia), in collaborazione con l'Università di Milano-Bicocca e lo spin-off "Bambini Bicocca", al fine di capire la vita quotidiana dei bambini e delle bambine di età compresa tra 1 e10 anni residenti nelle Province della Lombardia nell'emergenza pandemica. I dati emersi presentano «una fotografia coraggiosa della vita dei bambini in famiglia e una buona tenuta sistemica delle famiglie» (Mantovani et al., 2020, p. 7).

Dalla ricerca, oltre a una fotografia tracciata dai genitori su vissuti, risorse e fatiche dei bambini e delle bambine durante il lockdown, emerge che 
i servizi per l'infanzia (Nidi e Scuole dell'infanzia) sembrano aver mantenuto nella stragrande maggioranza un contatto stabile (l' $84,5 \%$ dei bambini $1-5$ anni frequentava il nido/scuola dell'infanzia e, di questi, il $72,3 \%$ ha avuto molti contatti con il Nido e la Scuola durante il lockdown). [...] Le proposte educative e didattiche sono state percepite dalle famiglie come mediamente "positive" $(39,6 \%)$, mentre il $41,5 \%$ dei genitori non si esprime in merito ("non so") e solo il $19 \%$ pensa siano state esperienze "negative". Il giudizio delle famiglie sulle proposte educative a distanza è, dunque, complessivamente buono (ivi, p. 21).

Da tale ricerca emergono dati altrettanto positivi anche in merito alla $\mathrm{DAD}$, che ha coinvolto i bambini e le bambine più grandi, e - relativamente alla relazione con le insegnanti - emerge che agli occhi del 42,1 $\%$ dei genitori esse non sono state sufficientemente presenti (Ibidem, passim).

Infine, pare opportuno segnalare che, nell'ambito della bozza di Linee pedagogiche per il sistema integrato "zerosei" promossa alla fine del 2020 dalla Commissione nazionale per il Sistema integrato di educazione e di istruzione (art. 10 del decreto legislativo 13 aprile 2017, n. 65), vi è un rinnovato invito a investire sulla coeducazione: le famiglie sono nuovamente valorizzate come partner fondamentali di un'alleanza educativa cruciale, al centro della quale vi sono la cura, il benessere e l'educazione di ciascun bambino e di ciascuna bambina che impegnano tutta la società in una responsabilità collettiva:

Educatori, insegnanti e genitori sono chiamati a confrontarsi con atteggiamento collaborativo e di rispetto reciproco, perché solo dalla coerenza educativa tra tutte le figure adulte che circondano il bambino può scaturire un percorso formativo che ne prenda in carico tutti gli aspetti: emotivi, cognitivi, relazionali, affettivi, morali, sociali. D'altra parte il nido e la scuola dell'infanzia rappresentano il primo contesto sociale allargato in cui il bambino si confronta con pari e adulti diversi, con regole e valori che possono non coincidere perfettamente con quelli già sperimentati: la conoscenza reciproca tra genitori e personale educativo, il dialogo aperto e improntato all'ascolto e all'accoglienza, la co-progettazione degli ambienti e dei percorsi educativi sono momenti concreti di una alleanza educativa, che sa rispettare le reciproche responsabilità. In questa dimensione sociale, che coinvolge bambini e adulti, i servizi educativi e le scuole dell'infanzia contribuiscono all'affermazione di una rinnovata idea di infanzia e dei suoi diritti, costruita con i soggetti protagonisti della relazione educativa, danno vita a reti sociali che adempiono a un'importante funzione di coesione e inclusione, proponendosi come punto di riferimento per il territorio di cui sono parte" (MIUR, 2020b, p.14). 
2. Breve presentazione della ricerca

\subsection{Obiettivi dell'indagine e aspetti metodologici}

Entro tale prospettiva si inserisce il percorso di ricerca esplorativa di seguito presentato e che, in questo contributo, vede un approfondimento specifico sul tema della relazione con le famiglie promossa da parte dei servizi 0-6 durante il lockdown.

La ricerca, di tipo esplorativo, attraverso un approccio misto qualiquantitativo (Trinchero, Robasto, 2019) si è proposta di indagare le modalità attraverso cui educatori, insegnanti e coordinatori pedagogici di servizi educativi per l'infanzia 0-6 in Italia hanno vissuto il periodo del lockdown e hanno riorganizzato la propria azione educativa e didattica sul piano della relazione educativa, delle proposte, delle modalità di coinvolgimento, della formazione, della progettazione e della documentazione.

Si è proceduto alla costruzione di un questionario strutturato con risposte chiuse ed aperte somministrato ad un campione di comodo, e collocato con accesso anonimo attraverso internet, dal titolo Educare a distanza, educare nell'emergenza.

Il questionario è formulato con 16 domande a risposta multipla, 8 su scala Likert a livelli (con ancoraggio variabile ma sempre dichiarato); sono presenti inoltre 18 domande a risposta aperta. Il questionario viene introdotto da un breve testo di presentazione che ne dichiara obiettivi e titolarità. Seguono quindi l'indicazione dell'anonimato delle risposte e l'utilizzo esclusivo ai fini della ricerca e nel rispetto della privacy, oltre alla comunicazione che gli esiti della ricerca saranno resi pubblici anche attraverso pubblicazioni open access. Il tempo di compilazione è di circa 30 minuti. Una sezione specifica del questionario è dedicata ai bisogni e alle richieste formulate dalle famiglie ad insegnanti ed educatori e ad aspetti di riorganizzazione del servizio attivati.

\subsection{Il campione e alcuni elementi contestuali}

Il questionario è stato reso accessibile online a partire dai primi giorni del mese di giugno 2020 e la raccolta si è conclusa a fine luglio 2020.

${ }^{6}$ Due sono i contributi presentati a partire da questa ricerca con approfondimenti differenti. 
I soggetti rispondenti sono stati 412 , in prevalenza di genere femminile $(98,3 \%$ ), di età media di 39,8 anni. Il 49,3\% di chi ha risposto al questionario dichiara di possedere una o più lauree in ambito pedagogico, 1 ' $8,8 \%$ lauree in altri settori, il $43,2 \%$ possiede il diploma di maturità. Il campione di rispondenti dichiara di lavorare per quasi una metà in un servizio di nido d'infanzia $(44,2 \%)$, per un'altra metà nella scuola dell'infanzia $(46,4 \%)$, residuale la presenza di chi opera servizi $0-6(13,3 \%)^{7}$. Si tratta di educatori di nido $(38,8 \%)$, insegnanti di scuola dell'infanzia $(35,9 \%)$ e funzioni di coordinamento della scuola $(20,3 \%)$ e altre figure educative $(5 \%)$. I rispondenti si distribuiscono tra appartenenti in servizi comunali $(29,9 \%)$, in quelli paritari $(27,7 \%)$, in privati $(20,1 \%)$ e nelle scuole statali $(15,8 \%)$. La collocazione geografica dei servizi dei rispondenti indica una netta prevalenza della Lombardia (59\%) ed Emilia-Romagna (23,5\%), seguite da Veneto, Piemonte, Lazio, Toscana e altre regioni in misura ridotta.

\section{Analisi dei dati: il focus sulle famiglie}

I rispondenti si sono attivati durante il lockdown con proposte che combinavano modalità in differita e diretta (ovvero sincrona) $(67,2 \%)$; un terzo ha preferito in modo esclusivo le modalità in differita $(28,6 \%)$; una percentuale ridotta in diretta $(3,6 \%)$ e in rari casi con nessuna modalità $(0,5 \%)$. Gli strumenti utilizzati in modo consistente dai rispondenti per comunicare le proprie proposte educative alle famiglie e ai bambini vedono una netta preferenza per Whatsapp o Telegram (72,8\%), seguiti da piattaforme per incontri $(66,3 \%)$. Viene poi segnalato l'impiego del telefono (43,2\%), della mail (40,1\%), l'uso di Facebook e affini $(30,1 \%)$, il Padlet $(25,9 \%)$ e il blog della scuola (20,6\%). Tra i formati utilizzati nell'ambito delle proposte educative viene segnalato in modo quasi preponderante l'uso dei video $(96,1 \%)$, seguito da foto $(80,3 \%)$, audio $(73,1 \%)$ e testi $(68,2 \%)$.

Tra gli obiettivi prioritari delle proposte educative offerte da parte dei servizi e delle scuole per l'infanzia, indicati da ciascun rispondente nel numero massimo di tre, vengono segnalati come prioritari i seguenti: favorire sollievo psicologico e benessere emotivo nei bambini $(65,3 \%)$, favorire momenti insieme tra bambini e figure familiari adulte $(56,6 \%)$,

${ }^{7}$ Erano previste più opzioni di risposta. 
suggerire proposte di gioco per i bambini $(55,8 \%)$ e favorire sollievo psicologico e benessere emotivo alle famiglie $(48,5 \%)$.

Ulteriori obiettivi indicati sono quelli relativi al promuovere apprendimenti dei bambini $(36,2 \%)$, al favorire la socialità con i compagni di sezione $(30,8 \%)$, ad offrire suggerimenti su pratiche di cura/autonomia dei bambini $(28,9 \%)$, al favorire un confronto con le figure famigliari adulte $(26,9 \%)$ e agli scambi tra le figure famigliari $(12,9 \%)$. Per quanto riguarda il feedback fornito dalle insegnanti ed educatrici ai bambini e relative famiglie in relazione alle proposte educative e didattiche, nel $49,8 \%$ è stato collettivamente in differita, nel $32 \%$ collettivamente in diretta, nel 36,7\% individualmente in differita, nel 18,9\% individualmente in diretta e nel 10,9\% non è stato dato.

Dalle risposte degli intervistati, risulta evidente come la disponibilità a contatti orali o scritti liberamente fruibili - da parte delle figure familiari - con tempi variabili sia stata una delle pratiche maggiormente adottate per sostenere le famiglie (settimanalmente dal $67,2 \%$ del campione, ogni due settimane dal $7 \%$, ogni mese dal $5,3 \%$ o ogni due mesi dal $3,4 \%)$.

Sono stati organizzati nel $55,1 \%$ dei servizi dei rispondenti incontri con le famiglie della sezione, nel 53,4\% dei casi colloqui individuali con alcune famiglie, nel 39,3\% colloqui con tutte le famiglie della sezione, nel 36,4\% incontri con tutte le famiglie del servizio.

Percentuali rilevanti di servizi non hanno calendarizzato mai momenti rivolti a tutte le famiglie del servizio $(63,6 \%)$, né colloqui individuali con le famiglie di tutti bambini $(60,7 \%)$, così come colloqui individuali con alcune famiglie $(46,6 \%)$, o incontri con le famiglie della sezione $(44,9 \%)$.

Tra le tre richieste più rilevanti ricevute da parte delle famiglie si evidenzia quella dell'aiuto per la gestione di difficoltà specifiche dei bambini $(58,7 \%)$, indicazioni per la gestione di specifiche pratiche di cura e autonomia dei bambini $(42,2 \%)$, sostegno sul piano personale $(38,6 \%)$, attività specifiche per i bambini differenti rispetto a quelle proposte prima del lockdown (32,2\%), aggiornamento sugli operatori e sul servizio $(28,4 \%)$, segnalazione di risorse $(27,7 \%)$, supporto in ordine alle nuove tecnologie $(10 \%)$, nessuna richiesta $(12,9 \%)$.

Il coinvolgimento da parte delle famiglie mostra un numero medio di 7,2 famiglie per sezione - se si rilevano i dati dei soli educatori ed insegnanti - che non hanno partecipato alle proposte; rispetto a queste famiglie non coinvolte il $67,1 \%$ si è poi attivato per sostenerne la partecipazione. 
L'assenza di coinvolgimento secondo i rispondenti è da imputare in modo determinante a difficoltà organizzative famigliari $(65,3 \%)$, a difficoltà emotive correlate all'emergenza $(43 \%)$, a condizioni di svantaggio di varia natura $(39,3 \%)$, a problemi economici dettati dalla situazione pandemica $(25,5 \%)$, ad una condizione di bisogno educativo speciale del bambino $(17,7 \%)$ e a difficoltà di salute legate all'epidemia in corso $(14,6 \%)$.

Tra i punti di forza individuati da parte dei rispondenti rispetto alle proposte educative in diretta emergono - rispetto al focus qui indagato la partecipazione con i genitori e con altre figure familiari tra cui i nonni, un mantenimento e talora un miglioramento della comunicazione (anche visiva e verbale) con le famiglie e una nuova vicinanza umana ed educativa nutrita di coinvolgimento emotivo e di un concreto fare insieme.

Tra i punti di debolezza emergono - insieme alle molte difficoltà tecniche, tecnologiche e di connessione e alla diffusa fatica di coinvolgere in modo armonico i bambini incontrati in diretta - anche alcuni aspetti specifici relativi alla relazione con le famiglie, tra cui: difficoltà a comprendere le fatiche delle famiglie, una resistenza di alcune famiglie a partecipare alle proposte in diretta insieme ai loro bambini sia per motivi organizzativi e di conciliazione con gli impegni familiari e professionali sia per una valutazione di inadeguatezza della proposta per l'età dei propri bambini ritenuti troppo piccoli e poco interessati.

Da una prima analisi delle risposte aperte relative alle richieste formulate dalle famiglie di cui educatori ed insegnanti hanno tenuto conto nel proprio lavoro, emergono alcuni nodi su cui riflettere. Le richieste della famiglie si possono innanzitutto distinguere in esigenze riguardanti le proposte rivolte ai bambini, quelle collegate a bisogni specifici delle famiglie e altre correlate ad una triangolazione genitori, bambini, insegnanti/educatori.

Per quanto riguarda le richieste relative alle proposte destinate ai bambini i suggerimenti dei genitori segnalano aspetti inerenti il contenuto delle proposte (ad es. necessità di letture, proposte ludiche o didattiche, anche in relazione alle età diverse dei bambini) o le modalità di comunicazione adottate nella dimensione della DAD (ad es. necessità di contatto visivo, richiesta di videochiamate, o videoincontri).

I bisogni formulati dai genitori hanno a che vedere con richieste di interventi/azioni rivolte al sostegno emotivo nella fase del lockdown agli adulti stessi (ad es. incontri individuali o collettivi per i genitori, incontri tematici, momenti di ascolto), all'accompagnamento alla genitorialità (ad es. gestione di situazioni vissute come problematiche, aiuto concreto nel- 
la quotidianità di gestione del bambino...), all'aver tenuto conto dei problemi organizzativi delle famiglie per una riorganizzazione flessibile delle proposte, così come un supporto all'impiego delle nuove tecnologie.

Si rintracciano anche richieste dei genitori volte alla costruzione di un legame tra genitori, bambini e insegnanti/educatori nella prospettiva di costruzione di un legame educativo di cura e fiducia, che si fonda su un esplicito riconoscimento della professionalità degli insegnanti ed educatori (ad es. condivisione delle scelte per lo sviluppo del bambino, costruzione di una routine che tenesse conto della continuità serviziocasa, feedback alle proposte).

\section{Interpretazione dei dati}

Coloro che hanno risposto sono apparsi fortemente, sebbene non uniformemente, reattivi, mostrando con ciò di aver avvertito la necessità di individuare forme di relazione con le famiglie capaci di accorciare precocemente e adeguatamente le distanze fisiche, emotive ed esperienziali generate dal lockdown. La pandemia ha colto impreparati, ma la percentuale che dichiara di aver ricercato strategie di comunicazione a supporto della relazione testimonia una propensione intrinseca, costitutiva di un ampio numero di servizi educativi, a individuare con ciascuna famiglia modi buoni di relazione nella quotidianità educativa (Guerra, Luciano, 2009) finanche pratiche di ascolto e risposte possibili anche nell'emergenza.

Ne sono prova, nell'ambito della ricerca, l'elevato numero di educatori, insegnanti e coordinatori che racconta di aver attivato da molto presto modalità di contatto, in una continua ricerca di dialogo e di condivisione in cui persistere anche nella consapevolezza della fatica e delle criticità che essa inevitabilmente comporta, oltre l'ovvietà (Bove, 2020): l'eterogeneità delle modalità, prevalentemente in forma mista, sembra indicare la sperimentazione di una strategia esplorativa attraverso la quale far emergere i canali e gli strumenti più idonei, ma contemporaneamente mostra un'attitudine, uno stile, una professionalità capaci di declinarsi flessibilmente anche in una situazione assolutamente inedita e addirittura inimmaginabile precedentemente.

I canali di comunicazione e gli strumenti utilizzati nei mesi del primo lockdown sono variegati, ad ulteriore sostegno di una modalità di procedere esplorativa. Tra questi, emergono in larga misura veicoli di comunicazione, come ad esempio Whatsapp, precedentemente utilizza- 
ti in gruppi distinti di educatori, insegnanti e coordinatori da un lato e di famiglie dall'altro. L'evento pandemico rivela una disponibilità a utilizzare strumenti e canali maggiormente dedicati ad un uso personale anche per comunicare con le famiglie, privilegiando probabilmente in tal senso la possibilità di raggiungere gli interlocutori nel modo per loro più agevole e rapido rispetto al mantenimento di confini netti tra personale e professionale. Sdoganare alcuni strumenti ritenuti propri della sfera privata è una suggestione a rompere alcune separazioni nette e con ciò a cogliere delle opportunità, mostrando finalmente l'assenza di una correlazione diretta sulla percezione dell'autorevolezza dei relativi ruoli: il passaggio a strumenti più personali, infatti, non sembra essere stato considerato un limite nel mantenimento dei rispettivi ruoli, professionale uno e genitoriale l'altro, ma valorizzato per l'immediatezza che ha potuto promuovere nelle prime comunicazioni.

Accanto a questi e secondariamente compaiono piattaforme per la comunicazione di gruppo scoperte in progress, seguite da "muri" nuovi come ad esempio Padlet, a suggerire una disponibilità alla sperimentazione di strumenti inediti.

Sempre alla propensione alla relazione connaturata nei professionisti educativi di questi servizi è attribuibile la ricerca di contatto con le famiglie meno o per nulla rispondenti, rispetto alle quali molti educatori, insegnanti e coordinatori sembrano abbastanza impegnati. Ciò ricolloca la scelta di servizi e scuole dell'infanzia di investire sull'alleanza educativa con le famiglie in una logica di partecipazione e democrazia che dà vita a reti sociali che adempiono alla funzione di coesione e inclusione nel territorio di cui i servizi e le famiglie sono parte, coerentemente con quanto suggerito dalla bozza di Linee guida pedagogiche 0-6 (MIUR, 2020b).

Del resto, uno sguardo di cura verso i bambini, il sostegno al gioco tra questi e i loro genitori e il benessere delle stesse famiglie risultano come le motivazioni più forti all'intervento nel periodo del lockdown, supportando l'immagine di professionisti fortemente orientati alla cura delle relazioni o, come poi è stato suggerito, dei legami educativi, non solo nei confronti dei bambini, ma anche delle loro famiglie, oltre che come esplicitamente emerge dagli obiettivi dichiarati dai rispondenti in merito alle proposte educative a distanza - nei confronti delle relazioni intra-familiari.

Il mantenimento in molti dei servizi di pratiche di relazione consuete pre-pandemia e lockdown come gli incontri con gruppi di famiglie o i colloqui individuali, in particolare per le condizioni percepite come di maggior fragilità, sostiene questa lettura, testimoniando uno sguardo at- 
tento e dedicato da parte di educatori, insegnanti e coordinatori anche agli adulti familiari e alle relazioni tra loro. In tal senso, pur nella fatica, i servizi e le scuole dell'infanzia non sembrano aver rinunciato a quel ruolo di promotori della cultura dell'infanzia, dell'educazione e della cura all'interno di strutture che sul territorio possono rendersi forum pubblici della società civile in cui esercitare la democrazia (Dahlberg, Moss, Pence, 1999, trad. it. 2003).

La diffusa disponibilità a contatti orali o scritti liberamente fruibili - da parte delle figure familiari - con tempi variabili, da un lato, e la proposta non capillare di incontri (individuali o collettivi) con le famiglie, dall'altro, se rapportati alla diffusa scelta di offrire proposte educative a distanza con l'obiettivo prioritario di fornire supporto psicologico e occasioni di gioco ai bambini e favorire relazioni con le loro figure familiari, costituiscono fattori che sembrano altresì suggerire che in diversi casi la scelta di scuole e servizi sia andata nella direzione di individualizzare il rapporto con le famiglie, individuando di volta in volta soluzioni e possibilità di comunicazione sulla base della contingenza delle singole realtà familiari.

Contemporaneamente, le richieste attribuite ai genitori sembrano riconoscere un ruolo determinante dei servizi educativi sia relativamente all'esperienza di crescita e apprendimento dei propri bambini, sia nel supporto alle loro stesse figure genitoriali, evidenziando come sia stato possibile attivare, laddove forse il terreno relazionale era già stato nutrito, una reciprocità di sguardi che ha sostenuto e alimentato proficuamente la progettualità di insegnanti ed educatori, pronti a cogliere, raccogliere e trasformare in prospettiva pedagogica la relazione educativa a distanza.

\section{Riflessioni conclusive}

Educatori, insegnanti e coordinatori che hanno risposto al questionario su base volontaria sono probabilmente rappresentativi di quella parte di servizi educativi $0 / 6$ che ha maggiormente scelto di lasciarsi interrogare dall'evento pandemico e dalle sue ripercussioni in termini di distanza, vicinanza, relazione anche relativamente alle famiglie, ma anche di quella parte di istituzioni educative alla ricerca di soluzioni, strategie, possibilità, nel presente e per il futuro. In questo senso, le risposte non possono essere generalizzate, a campioni più estesi, eppure suggeriscono aperture utili da osservare. 
Sicuramente non rappresenta una variabile ininfluente il fatto che oltre l'$^{\prime} 80 \%$ delle risposte provenga da operatori di due tra le Regioni maggiormente colpite durante la prima ondata pandemica e che quasi il $60 \%$ riguardi la Lombardia, Regione che ha subito il maggior numero di perdite e che nella prima ondata è stata il territorio più martoriato. La precocità delle chiusure e delle perdite ha probabilmente influito su un altrettanto precoce attivazione per rispondere al lockdown e alla relativa chiusura dei servizi educativi, scegliendo di impegnarsi nell'attivazione di strategie di comunicazione e ripresa delle relazioni. Il riconoscimento dei propri bisogni, in quella fase, ha sollecitato forse una attenzione ai bisogni altrui, compresi quelli delle famiglie. Se ciò non riguarda la totalità dei servizi, certamente i dati raccolti indicano una diffusa propensione a tenere aperto, a riattivare e a rinnovare continuamente il dialogo con le famiglie - come del resto auspicato nell'ambito degli approcci partecipati dalle relazioni nei servizi 0-6 (Bondioli, Savio 2018) e delle recenti Linee pedagogiche per il sistema integrato "zerosei" (MIUR, 2020b) - esplorando modalità inedite accanto a quelle più note, rompendo tabù, $\mathrm{ad}$ esempio relativamente a canali personali o strumenti digitali, e mostrando ulteriormente la preziosità della relazione con le famiglie, sia come anello di congiunzione nella relazione con i bambini, sia come pari con cui costruire una relazione a beneficio dei bambini come degli adulti.

In tal senso, la scelta del personale educativo dei servizi e delle scuole dell'infanzia di persistere nell'atroce difficoltà del lockdown per coprogettare pratiche e proposte finalizzate a rinsaldare legami con le famiglie e con i bambini mostra l'adesione a un progetto di resilienza che, come usualmente accade nella prospettiva della pedagogia dell'emergenza, dedica cura e attenzione sia verso gli individui sia verso le comunità, rispondendo pienamente ad un'idea di militanza pedagogica che, attraverso una progettualità di tipo educativo, si orienta verso nuovi equilibri individuali, comunitari e sociali (Vaccarelli, Mariantoni, 2018).

\section{Riferimenti bibliografici}

Antonietti, M., M. Guerra, and E. Luciano (2020a: Trasformare la relazione educativa nelle situazioni di emergenza: Un'indagine nei servizi 0-6 al tempo del Covid-19. Pedagogia Oggi, XVIII (2), pp. 63-75.

Antonietti, M., M. Guerra, and E. Luciano (2020b): Servizi 0/6 nell'emergenza e famiglie con bambini/e in condizione di bisogno educativo speciale: prove di relazione da un'indagine in tempi di pandemia. Nuova Secondaria, (2), pp. 365-378. 
Bondioli A., Savio D. (2018): Educare l'infanzia. Temi chiave per i servizi 0-6. Roma: Carocci.

Bove C. (2003): Riflessioni sull'evoluzione culturale e professionale del nido. In L. Gandini, S. Mantovani, C.P. Edwards (a cura di): Il nido per una cultura dell'infanzia. Azzano San Paolo (Bg): Junior.

Bove C. (2020): Capirsi non è ovvio. Dialogo tra insegnanti e genitori in contesti educativi. Milano: FrancoAngeli.

Bronfenbrenner U. (1979): The Ecology of Human Development: Experiments by Nature and Design. Cambridge, Massachusetts: Harvard University Press. Trad. it. Bologna: Il Mulino, 1986.

Brougère G. (2009): Une théorie de l'apprentissage adaptée: l'apprentissage comme participation. En G. Brougère, A.L. Ulmann (dir.): Apprendre de la vie quotidienne. Paris: PUF.

Cagliari P. (2013): La partecipazione sostanzia i diritti. Children in Europe, $\mathrm{n}$. 24, pp. 6-7.

Campioni L., Marchesi F. (a cura di) (2014): Sui nostri passi. Tracce di storia dei servizi educativi nei Comuni capoluogo dell'Emilia-Romagna. Parma: Edizioni Junior-Spaggiari Edizioni.

Commissione Europea (2016): Un quadro europeo per la qualità dei servizi educativi e di cura per l'infanzia: proposta di principi chiave. Gruppo di lavoro tematico sull'educazione e cura dell'infanzia (trad. it. a cura di A. Lazzari). Bergamo: ZeroseiUp.

Corsi M. (2020): Il dopo delle famiglie: disagi, quasi certezze e speranze. RIEFRivista Italiana di Educazione Familiare, just accepted.

Cyrulnik B. (2005): Abbandono e tutori di resilienza. In B. Cyrulnik, E. Malaguti, (a cura di): Costruire la resilienza. La riorganizzazione positiva della vita e la creazione di legami positivi. Trento: Erickson.

Dahlberg G., Moss P., Pence A.R. (1999): Oltre la qualità nell'educazione e cura della prima infanzia. I linguaggi della valutazione. Trad. it. Reggio Emilia: Reggio Children, 2003.

Edwards C., Gandini L., Forman G., (a cura di) (1995): I cento linguaggi dei bambini. Bergamo: Junior.

Fielding M., Moss P. (2010): Radical education and the common school. A democratic alternative. London: Routledge. Trad. it. Parma: Junior-Spaggiari, 2014.

Gigli A. (2020), Essere genitori ai tempi del Covid-19: disagi, bisogni, risorse: i primi dati di una rilevazione. RIEF-Rivista Italiana di Educazione Familiare, just accepted.

Gigli A. (a cura di) (2020): Infanzia, famiglie, servizi educativi e scolastici nel Covid-19. Riflessioni pedagogiche sugli effetti del lockdown e della prima fase di riapertura. CENTRO RICERCHE EDUCATIVE SU INFANZIE E FAMIGLIE CREIF, Dipartimento di Scienze dell'educazione "G.M. Bertin" dell'Alma Mater Studiorum - Università di Bologna, 2020 (https://edu.unibo.it/it/terza-missione/formazione/dossier-creif; data di ultima consultazione: 30.11.20). 
Gigli A. (a cura di) (2021): Oltre l'emergenza. Sguardi pedagogici su infanzia, famiglie, servizi educativi e scolastici nel Covid-19. Parma: Junior-Spaggiari.

Guerra M., Luciano E. (2009): La relazione con le famiglie nei servizi e nelle scuole per l'infanzia. Bergamo: Junior.

Guerra M., Luciano E. (a cura di) (2015): Costruire partecipazione. La relazione tra famiglie e servizi per l'infanzia in una prospettiva internazionale. Parma: Junior-Spaggiari.

Harris A., Goodall J. (2006): Parental Involvement in Education: An overview of the Literature. Coventry (UK): University of Warwick.

Lazzari A. (2016): I servizi per l'infanzia oltre la crisi: un impegno politico e pedagogico. In: Commissione Europea: Un quadro europeo per la qualità dei servizi educativi e di cura per l'infanzia: proposta di principi chiave. Gruppo di lavoro tematico sull'educazione e cura dell' infanzia. Bergamo: ZeroseiUp, pp. 5-16.

Luciano, E., Marcuccio M. (2017): Famiglie e servizi: verso una tipologia di forme di partecipazione nei servizi per l'infanzia. RIEF-Rivista Italiana di Educazione Familiare, n. 1, pp. 123-143.

Malaguti E. (2005): Educarsi alla resilienza. Come affrontare crisi e difficoltà e migliorarsi. Trento: Erickson.

Mantovani S., Picca M., Ferri P., Bove C., Manzoni P. (2020): Bambini e lockdown. La parola ai genitori. Milano: Università degli Studi Milano-Bicocca.

Milani P. (a cura di) (2008): Co-educare i bambini. Bari: Pensa Multimedia.

Milani P. (2018): Educazione e famiglie. Ricerche e nuove pratiche per la genitorialità. Roma: Carocci.

MIUR (2020a): Orientamenti pedagogici sui Legami educativi a Distanza. Un modo diverso per "fare" nido e scuola dell'infanzia - Commissione nazionale per il sistema integrato zerosei (D.lgs. 65/2017).

MIUR (2020b): Linee pedagogiche per il sistema integrato "zerosei" - Commissione nazionale per il sistema integrato zerosei (D.lgs. 65/2017).

OECD (2001): Starting Strong I. Early Childhood Education and Care. Paris: OECD Publishing.

OECD (2006): Starting Strong II. Early Childhood Education and Care. Paris: OECD Publishing.

OECD (2011): Starting Strong III. A Quality Toolbox for Early Childhood Education and Care. Paris: OECD Publishing.

OECD (2015): Starting Strong IV. Monitoring Quality in Early Childhood Education and Care. Paris: OECD Publishing.

OECD (2017): Starting Strong V. Monitoring Quality in Early Childhood Education and Care. Paris: OECD Publishing.

Palmieri C. (2000): La cura educativa. Riflessioni ed esperienze tra le pieghe dell'educare. Milano: FrancoAngeli.

Pastori G., Mangiatordi A., Pagani V., Pepe A. (2020): Che ne pensi? La didattica a distanza dal punto di vista dei genitori. Milano: Università degli Studi Milano-Bicocca (https://bit.ly/report-dad; data di ultima consultazione: 30.1.21). 
Pileri A. (2017): Co-educare attraverso una prospettiva inclusiva. Il progetto RICE a Treviso. Studium Educationis, n. 3, pp. 55-64.

Pileri A. (2020): Infanzie, famiglie, personale dei servizi prescolari al tempo del Covid-19. Quale co-educazione possibile?. In A. Gigli (a cura di): Infanzia, famiglie, servizi educativi e scolastici nel Covid-19. Riflessioni pedagogiche sugli effetti del lockdown e della prima fase di viapertura, cit., pp. 80-86. (https:// edu.unibo.it/it/terza-missione/formazione/dossier-cref; data di ultima consultazione: 30.11 .20$)$.

Pourtois J.-P., Desmet H. (dir.) (2015): L'éducation émancipatrice. De la co-education école-famille à la Cité de l'éducation. Paris: Education et Formation PUF.

Powell D.R., Son S., File N., San Juan R.R. (2010): Parent-School Relationships and Children's Academic and Social Outcomes in Public School Pre-Kindergarten. Journal of School Psychology, n. 48, pp. 269-292.

Rayna S., Rubio M.N., Scheu H. (dir.) (2010): Parents-professionnels: la coéducation en questions. Toulouse: Erès.

Trinchero R., Robasto D. (2019): I mixed methods nella ricerca educativa. Milano: Mondadori.

Vaccarelli A., Mariantoni S. (2018): Individui, comunità e istituzioni in emergenza. Intervento psico-socio-pedagogico e lavoro di rete nelle situazioni di catastrofe. I territori dell'educazione. Milano: FrancoAngeli.

Weiss H., Caspe M., Lopez M.E. (2008): Family Involvement Promotes Success for Young Children: A Review of Recent Research. In M.M. Cornish (ed.): Promising Practices for Partnering with Families in the Early Years. Plymouth: Information Age Publishing. 